\title{
ON SLANT CURVES IN SASAKIAN 3-MANIFOLDS
}

\author{
Jong TaEk Cho, Jun-IChi Inoguchi and Ji-Eun LeE
}

A classical theorem by Lancret says that a curve in Euclidean 3-space is of constant slope if and only if its ratio of curvature and torsion is constant. In this paper we study Lancret type problems for curves in Sasakian 3-manifolds.

\section{INTRODUCTION}

In classical differential geometry of spatial curves, the following result is known (see for example, $[10,19,21])$.

ThEOREM 1.1. (Bertrand-Lancret-de Saint Venant) $A$ curve $\gamma(s)$ in Euclidean 3-space $\mathbb{E}^{3}$ is a curve of constant slope if and only if its ratio of curvature and torsion is constant.

Here we recall that a curve in $\mathbb{E}^{3}$ is said to be a curve of constant slope (or cylindrical helix [20]) if the tangent vector field of $\gamma$ has constant angle with a fixed direction (called the axis of the curve). Moreover it is clear that for every curve $\gamma$ of constant slope, there exists a cylinder on which $\gamma$ moves in such a way as to cut each ruling at a constant angle. (See [20, pp. 72-73].)

Barros [1] generalised the above characterisation due to Bertrand-Lancret-de Saint Venant to curves in 3-dimensional space forms. Corresponding results for 3-dimensional Lorentzian space forms are obtained by Ferrández [11]. Moreover Ferrández, Giménez and Lucas $[12,13]$ investigated Bertrand-Lancret-de Saint Venant problem for null curves in Minkowski 3-space. (See also [14, 18].)

As is well known, the unit 3-sphere $S^{3}$ is a typical example of a Sasakian manifold. In 3-dimensional contact metric geometry, Legendre curves play a fundamental role [2]. As a generalisation of Legendre curves, in this paper, we introduce the notion of a slant curve.

A curve in a contact 3-manifold is said to be slant if its tangent vector field has constant angle with the Reeb vector field. Slant curves appear naturally in differential geometry of Sasakian 3-manifolds. In our recent paper [9], it is shown that biharmonic curves in 3-dimensional Sasakian space forms are slant helices.

Received 9th May, 2006

Copyright Clearance Centre, Inc. Serial-fee code: 0004-9727/06 \$A2.00+0.00. 
In this paper we study Bertrand-Lancret-de Saint Venant type problems for slant curves in Sasakian 3-manifolds.

Our result is

Theorem. A curve in a Sasakian 3-manifold is a slant curve if and only if its ratio of "geodesic curvature" and "geodesic torsion \pm 1 " is constant.

Moreover, we find the explicit parametric examples of proper slant curves which are not helices in the Heisenberg group $\mathbb{H}_{3}$ (see Example 4.2).

\section{Preliminaries}

2.1. Let $\gamma: I \rightarrow M=\left(M^{3}, g\right)$ be a Frenet curve parametrised by arc length in a Riemannian 3-manifold $M^{3}$ with Frenet frame field $(T, N, B)$. Here $T, N, B$ are the tangent, principal normal and binormal vector fields, respectively. Denote by $\nabla$ the LeviCivita connection of $(M, g)$. Then the Frenet frame satisfies the following Frenet-Serret equations:

$$
\nabla_{T} T=\kappa N, \quad \nabla_{T} N=-\kappa T+\tau B, \quad \nabla_{T} B=-\tau N
$$

where $\kappa=\left|\nabla_{T} T\right|$ and $\tau$ are the geodesic curvature and geodesic torsion of $\gamma$, respectively. A Frenet curve is said to be a helix if both of $\kappa$ and $\tau$ are constant.

2.2. Next, we recall the fundamental ingredients of 3-dimensional contact metric geometry. Our general reference is [3].

Let $M$ be a 3-dimensional manifold. A contact form is a one-form $\eta$ such that $d \eta \wedge \eta \neq 0$ on $M$. A 3-manifold $M$ together with a contact form $\eta$ is called a contact 3-manifold. The Reeb vector field $\xi$ is a unique vector field satisfying

$$
\eta(\xi)=1, d \eta(\xi, \cdot)=0
$$

On a contact 3-manifold $(M, \eta)$, there exists a structure tensor $(\varphi, \xi, g)$ such that

$$
\begin{gathered}
\varphi^{2}=-I+\eta \otimes \xi, \quad g(\varphi X, \varphi Y)=g(X, Y)-\eta(X) \eta(Y), \\
g(X, \varphi Y)=d \eta(X, Y), \quad X, Y \in \mathfrak{X}(M) .
\end{gathered}
$$

The structure $(\varphi, \xi, \eta, g)$ is called the associated contact metric structure of $(M, \eta)$. A contact 3-manifold together with its associated contact metric structure is called a contact metric 3-manifold. A contact metric 3-manifold $M$ satisfies the following formula ([22]).

$$
\left(\nabla_{X} \varphi\right) Y=g(X+\mathrm{h} X, Y) \xi-\eta(Y)(X+\mathrm{h} X), \quad X, Y \in \mathfrak{X}(M)
$$

where $\mathrm{h}=£_{\xi} \varphi / 2$. 
A contact metric 3-manifold $(M, \varphi, \xi, \eta, g)$ is called a Sasakian manifold if it satisfies

$$
\left(\nabla_{X} \varphi\right) Y=g(X, Y) \xi-\eta(Y) X
$$

for all $X, Y \in \mathfrak{X}(M)$.

A plane section $\Pi_{x}$ at a point $x$ of a contact metric 3-manifold is called a holomorphic plane if it is invariant under $\varphi_{x}$. The sectional curvature function of holomorphic planes is called the holomorphic sectional curvature. Sasakian 3-manifolds of constant holomorphic sectional curvature are called 3-dimensional Sasakian space forms. Simply connected and complete 3-dimensional Sasakian space forms are classified as follows:

Propos I T I ON 2.1. ([4]) Simply connected and complete 3-dimensional Sasakian space forms $\mathcal{M}^{3}(H)$ of constant holomorphic sectional curvature $H$ are isomorphic to one of the following unimodular Lie groups with left invariant Sasakian structures: the special unitary group $\mathrm{SU}(2)$ for $H>-3$, the Heisenberg group $\mathbb{H}_{3}$ for $H=-3$, or the universal covering group $\widetilde{\mathrm{SL}}(2, \mathbb{R})$ of the special linear group $\mathrm{SL}(2, \mathbb{R})$ for $H<-3$. The Sasakian space form $\mathcal{M}^{3}(1)$ is the unit 3-sphere $S^{3}$ with the canonical Sasakian structure.

\section{Slant curves}

3.1. Let $M$ be a contact metric 3-manifold and $\gamma(s)$ a Frenet curve parametrised by arc length $s$ in $M$. The contact angle $\theta(s)$ is a function defined by $\cos \theta(s)=g(T(s), \xi)$. A curve $\gamma$ is said to be a slant curve if its contact angle is constant. Slant curves of contact angle $\pi / 2$ are traditionally called Legendre curves. The Reeb flow is a slant curve of contact angle 0 .

Now we consider Bertrand-Lancret-de Saint Venant type results for contact geometry. We take an adapted local orthonormal frame field $\{X, \varphi X, \xi\}$ of $M$ such that $\eta(X)=0$.

Let $\gamma$ be a non-geodesic Frenet curve in a Sasakian 3-manifold. Differentiating the formula $g(T, \xi)=\cos \theta$ along $\gamma$, then it follows that

$$
-\theta^{\prime} \sin \theta=g(\kappa N, \xi)+g(T,-\varphi T)=\kappa \eta(N) .
$$

This equation implies the following result.

Proposition 3.1. A non-geodesic curve $\gamma$ in a 3-dimensional Sasakian manifold $M$ is a slant curve if and only if it satisfies $\eta(N)=0$.

Hence $T, N$ and $\xi$ of a slant curve $\gamma(s)$ has the form

$$
\begin{aligned}
T & =\sin \theta\{\cos \beta(s) X+\sin \beta(s) \varphi X\}+\cos \theta \xi, \\
N & =-\sin \beta(s) X+\cos \beta(s) \varphi X, \\
\xi & =\cos \theta T \pm \sin \theta B
\end{aligned}
$$


for some function $\beta(s)$. Differentiating $0=g(N, \xi)$ along $\gamma$ and using the Frenet-Serret equations, we have

$$
\kappa \cos \theta+(-1 \pm \tau) \sin \theta=0 .
$$

This implies that the ratio of $\tau \pm 1$ and $\kappa$ is a constant. Conversely, if the ratio of $\tau \pm 1$ and $\kappa \neq 0$ is constant, then $\gamma$ is clearly a slant curve. Thus we obtain the following result.

THEOREM 3.1. A non-geodesic curve in a Sasakian 3-manifold $M$ is a slant curve if and only if its ratio of $\tau \pm 1$ and $\kappa$ is constant.

The equation (3.1) implies the following result (compare with [2]).

COROLlaRY 3.1. Let $\gamma$ be a non-geodesic slant curve. Then $\tau= \pm 1$ if and only if $\gamma$ is a Legendre helix.

3.2. A Sasakian 3-manifold $M$ is said to be regular if its Reeb vector field $\xi$ generates a one-parameter group $K$ of isometries on $M$, such that the action of $K$ on $M$ is simply transitive. The Killing vector field $\xi$ induces a regular one-dimensional Riemannian foliation on $M$. We denote by $\bar{M}:=M / \xi$ the orbit space (the space of all leaves) of a regular Sasakian 3-manifold $M$ under the $K$-action.

The Sasakian structure on $M$ induces a Kähler structure on the orbit space $\bar{M}$. Further the natural projection $\pi: M \rightarrow \bar{M}$ is a Riemannian submersion. It is easy to see that $M$ is a Sasakian space form of constant $\varphi$-holomorphic sectional curvature $H$ if and only if $\bar{M}$ is a space form of curvature $H+3$.

Take a curve $\bar{\gamma}$ in the orbit space, then its inverse image $S_{\bar{\gamma}}=\pi^{-1}(\bar{\gamma})$ is a flat surface in $M$. This flat surface is called the Hopf cylinder over $\gamma$. The mean curvature of the Hopf cylinder is the half of the geodesic curvature of $\bar{\gamma}$.

In particular, if $M$ is the unit 3-sphere $S^{3}$, then $\pi$ coincides with the Hopf fibreing $S^{3}(1) \rightarrow S^{2}(4)$. In this case, if $\vec{\gamma}$ is a small circle, then its Hopf cylinder is a non-minimal constant mean curvature torus. If $\bar{\gamma}$ is a great circle, then its Hopf cylinder is the Clifford minimal torus.

Now we consider a slant curve $\gamma$ with the contact angle $\theta$ in a regular Sasakian 3-manifold. Let $\bar{\gamma}=\pi \circ \gamma$ be the projection of $\gamma$ onto $\bar{M}$. Direct computation shows that the arc length parameter $\bar{s}$ of $\bar{\gamma}$ is

$$
\bar{s}=\frac{s}{\sin \theta} .
$$

The Frenet frame $\{\bar{T}(\bar{s}), \bar{N}(\bar{s})\}$ of $\bar{\gamma}$ is given by

$$
\bar{T}(\bar{s})=\frac{1}{\sin \theta} \pi_{*} T(s), \quad \bar{N}(\bar{s})= \pm \pi * N(s) .
$$

Thus the signed curvature $\bar{\kappa}$ of $\bar{\gamma}$ is given by

$$
\bar{\kappa}(\bar{s})=\frac{ \pm 1}{\sin ^{2} \theta} \kappa(s) .
$$


We specialise the contact angle of slant curves. Let $\gamma(s)$ be a Legendre curve in a regular contact Riemannian 3-manifold $M$. Then from (3.2) we see that its projection $\bar{\gamma}(s)=\pi(\gamma(s))$ is a curve with arc length parameter $s$ and that $\gamma$ is a horizontal lift of $\bar{\gamma}$. Further, the signed curvature $\bar{\kappa}$ is given by $\bar{\kappa}(s)= \pm \kappa(s)$. We note that for the Hopf cylinder $S=\pi^{-1}(\bar{\gamma})$, the Reeb vector field $\xi$ is tangent to $S$ and $S$ contains $\gamma$.

\section{EXAMPLES AND REMARKS}

Let $M$ be a Riemannian 3-manifold and $\gamma$ a curve in $M$ parametrised by arc length. Then $\gamma$ is said to be biharmonic if

$$
\nabla_{T}^{3} T+R(\kappa N, T) T=\mathbf{0}
$$

Caddeo, Montaldo and Piu [5] classified biharmonic curves in the unit 3-sphere $S^{3}$. Caddeo, Piu and Oniciuc [7] classified biharmonic curves in the Heisenberg group. The present authors generalised the results of [7] to general 3-dimensional Sasakian space forms [9]. Caddeo, Montaldo Oniciuc and Piu generalised the classification of [9] to Bianchi-Cartan-Vranceanu spaces [6].

TheOREM 4.1. ([9]) Every proper biharmonic curve in Sasakian space form with constant holomorphic sectional curvature $H$ is a slant helix satisfying

$$
\kappa^{2}+\tau^{2}=1+(H-1) \sin ^{2} \theta
$$

Thus classification of proper biharmonic curves in a Sasakian 3-space form reduces to solving the equations:

$$
\kappa^{2}+\tau^{2}=1+(H-1) \sin ^{2} \theta, \kappa \cos \theta+(-1 \pm \tau) \sin \theta=0 .
$$

REMARK 1. Let $M$ be one of the following 3-dimensional spaces; Riemannian space form, or Minkowski 3-space. Then the biharmonic equation for non-geodesics in $M$ is given by the following:

(1) $M$ is of constant curvature $c$, then $\kappa=$ constant and $\kappa^{2}+\tau^{2}=c([5])$,

(2) $M$ is the Minkowski 3-space, then $\kappa=$ constant and $\kappa^{2}-\tau^{2}=0$ $([8,15,16])$.

EXAMPLE 4.1. ([7,9]) The Heisenberg group $\mathbb{H}_{3}$ is a Cartesian 3-space $\mathbb{R}^{3}(x, y, z)$ furnished with the group structure

$$
\left(x^{\prime}, y^{\prime}, z^{\prime}\right) \cdot(x, y, z)=\left(x^{\prime}+x, y^{\prime}+y, z^{\prime}+z+\left(x^{\prime} y-y^{\prime} x\right) / 2\right) .
$$

Define the left-invariant metric $g$ by

$$
g=\frac{d x^{2}+d y^{2}}{4}+\eta \otimes \eta, \quad \eta=\frac{1}{2}\left\{d z+\frac{1}{2}(y d x-x d y)\right\}
$$


We take a left-invariant orthonormal frame field $\left(e_{1}, e_{2}, e_{3}\right)$ :

$$
e_{1}=2 \frac{\partial}{\partial x}-y \frac{\partial}{\partial z}, e_{2}=2 \frac{\partial}{\partial y}+x \frac{\partial}{\partial z}, e_{3}=2 \frac{\partial}{\partial z}
$$

Then the commutation relations are derived as follows:

$$
\left[e_{1}, e_{2}\right]=2 e_{3},\left[e_{2}, e_{3}\right]=\left[e_{3}, e_{1}\right]=0
$$

The dual frame field $\left(\theta^{1}, \theta^{2}, \theta^{3}\right)$ is given by

$$
\theta^{1}=\frac{1}{2} d x, \theta^{2}=\frac{1}{2} d y, \theta^{3}=\frac{1}{2} d z+\frac{y d x-x d y}{4} .
$$

Then the 1-form $\eta=\theta^{3}$ is a contact form and the vector field $\xi=e_{3}$ is the Reeb vector field on $\mathbb{H}_{3}$.

We define a $(1,1)$-tensor field $\varphi$ by

$$
\varphi e_{1}=e_{2}, \varphi e_{2}=-e_{1}, \varphi \xi=0
$$

Then we find

$$
d \eta(X, Y)=g(X, \varphi Y)
$$

and hence, $(\eta, \xi, \varphi, g)$ is a contact metric structure. Moreover, we see that it becomes a Sasakian structure. Then every proper biharmonic curve in $\mathbb{H}_{3}$ is represented as

$$
\left\{\begin{array}{l}
x(s)=\frac{1}{A} \sin \theta \sin (A s+a)+b \\
y(s)=-\frac{1}{A} \sin \theta \cos (A s+a)+c \\
z(s)=\left(\cos \theta+\frac{\sin ^{2} \theta}{2 A}\right) s-\frac{b}{2 A} \sin \theta \cos (A s+a)-\frac{c}{2 A} \sin \theta \sin (A s+a)+d
\end{array}\right.
$$

for a constant contact angle $\theta$, where $A, a, b, c, d$ are constants. These slant helices satisfy $\kappa^{2}+\tau^{2}=1-4 \sin ^{2} \theta$. Note that in [7], the metric on $\mathbb{H}_{3}$ is chosen as $4 g$.

EXAMPLE 4.2. We construct a proper slant curve $\gamma$ which is not a helix in the above $\mathbb{H}_{3}$. Let $\gamma$ be a slant curve in $\mathbb{H}_{3}$. Then for a constant $\theta$ we put

$$
\gamma^{\prime}(s)=T(s)=T_{1} e_{1}+T_{2} e_{2}+T_{3} e_{3}
$$

and

$$
T_{1}(s)=\sin \theta \cos \beta(s), T_{2}=\sin \theta \sin \beta(s), T_{3}=\cos \theta .
$$

By using Frenet-Serret equations (2.1) we compute the geodesic curvature $\kappa$ and the geodesic torsion $\tau$ for a slant curve $\gamma$ in $\mathbb{H}_{3}$. Then we obtain

$$
\begin{aligned}
& \kappa=\sin \theta\left(\beta^{\prime}(s)-2 \cos \theta\right), \\
& \tau=\cos \theta\left(\beta^{\prime}(s)-2 \cos \theta\right)+1,
\end{aligned}
$$


where we assume that $\sin \theta\left(\beta^{\prime}(s)-2 \cos \theta\right)>0$.

Here, the tangent vector field $T$ of $\gamma$ is also represented by the following:

$$
T=\left(\frac{d x}{d s}, \frac{d y}{d s}, \frac{d z}{d s}\right)=\frac{d x}{d s} \frac{\partial}{\partial x}+\frac{d y}{d s} \frac{\partial}{\partial y}+\frac{d z}{d s} \frac{\partial}{\partial z} .
$$

Then it follows that

$$
\frac{d x}{d s}=2 T_{1}, \frac{d y}{d s}=2 T_{2}, \frac{d z}{d s}=2 T_{3}+\left(x \frac{d y}{d s}-y \frac{d x}{d s}\right) .
$$

In view of (4.3), we take for example $\beta(s)=\ln s$. Then we can find an explicit parametric equations of slant curves $\gamma$ which are not helices:

$$
\left\{\begin{array}{l}
x(s)=2 \sin \theta \cdot \frac{s}{2}\{\sin (\ln s)+\cos (\ln s)\}+b_{1} \\
y(s)=2 \sin \theta \cdot \frac{s}{2}\{\sin (\ln s)-\cos (\ln s)\}+c_{1} \\
z(s)=4\left(\frac{1}{4} \sin ^{2} \theta\right) s^{2}+2(\cos \theta) s+d_{1}
\end{array}\right.
$$

where $b_{1}, c_{1}, d_{1}$ are constants.

EXAMPLE 4.3. (Grassmann geometry) Let $M$ be a Riemannian manifold and $\mathrm{Gr}_{\ell}(T M)$ its Grassmann bundle of all $\ell$-planes in $T M(1 \leqslant \ell \leqslant \operatorname{dim} M)$. Take a non-empty subset $\Sigma$ of $\mathrm{Gr}_{\ell}(T M)$. An $\ell$-dimensional submanifold $\phi: S \rightarrow M$ of $M$ is said to be a $\Sigma$ submanifold of $M$ if $d \phi(T S) \subset \Sigma$. The collection of all $\Sigma$-submnaifolds is called the $\Sigma$-geometry of $M$. Grassmann geometry is a collected name for such a $\Sigma$-geometry. Let us denote by $G$ the identity component of the isometry group of $M$. Then $G$ naturally acts on $\mathrm{Gr}_{\ell}(T M)$. If $\Sigma$ is an $G$-orbit in $\mathrm{Gr}_{\ell}(T M)$, the $\Sigma$-geometry is called of orbit type.

In [17], Inoguchi, Kuwabara and Naitoh investigated the Grassmann geometry of orbit type in $\mathbb{H}_{3}$. In this case, the $G$-orbit spaces in $\mathrm{Gr}_{2}\left(T \mathbb{H}_{3}\right)$ are parametrised by the curvature function $K$ and $K$ takes value in the closed interval $[-3,1]$. The following results were obtained in [17]:

Proposition 4.1. For any $\alpha \in(-3,1), \mathcal{O}(\alpha)$-surfaces are of constant negative curvature $\alpha-1$.

THEOREM 4.2. For any $\alpha \in(-3,1)$,

(1) $\mathcal{O}(\alpha)$-surfaces are of constant negative curvature $\alpha-1$.

(2) there exist local $\mathcal{O}(\alpha)$-surfaces foliated by circles which are helices of $\mathbb{H}_{3}$ with the same curvature and torsion 1.

The helices on $\mathcal{O}(\alpha)$-surfaces are slant helices. In fact, the contact angle $\theta$ is computed as

$$
\cos \theta=-\sqrt{1-\rho^{2}}, \quad \rho:=\frac{1}{2} \sqrt{1-\alpha} .
$$

These helices have geodesic curvature $\kappa=2 \rho / \sqrt{1-\rho^{2}}$ and geodesic torsion $\tau=1$, and hence do not satisfy the relation $\kappa^{2}+\tau^{2}=1-4 \sin ^{2} \theta$. Thus these slant helices are non-biharmonic. 


\section{REFERENCES}

[1] M. Barros, 'General helices and a theorem of Lancret', Proc. Amer. Math. Soc. 125 (1997), 1503-1509.

[2] C. Baikoussis and D.E. Blair, 'On Legendre curves in contact 3-manifolds', Geom. Dedicata 49 (1994), 135-142.

[3] D.E. Blair, Riemannian geometry of contact and symplectic manifolds, Progress in Math. 203 (Birkhäuser, Boston, Basel, Berlin, 2002).

[4] D.E. Blair and L. Vanhecke, 'Symmetries and $\varphi$-symmetric spaces', Tôhoku Math. J. 39 (1997), 373-383.

[5] R. Caddeo, S. Montaldo and C. Oniciuc, 'Biharmonic submanifolds of $S^{3}$, Internat. J. Math. 12 (2001), 867-876.

[6] R. Caddeo, S. Montaldo, C. Oniciuc and P. Piu, 'The classification of biharmonic curves of Cartan-Vranceanu 3-dimesnional spaces', (preprint, math.DG/0510435), in Proceedings of The Seventh International Workshop on Differential Geometry and its Applications, Deva, Romania, September 2005 (to appear).

[7] R. Caddeo, C. Oniciuc and P. Piu, 'Explicit formulas for biharmonic non-geodesic curves of the Heisenberg group', Rend. Sem. Mat. Univ. Politec. Torino 62 (2004), 265-277.

[8] B.Y. Chen and S. Ishikawa, 'Biharmonic surfaces in pseudo-Euclidean spaces', Mem. Fac. Sci. Kyushu Univ. Ser A 45 (1991), 323-347.

[9] J.T. Cho, J. Inoguchi and J.-E. Lee, 'Biharmonic curves in Sasakian space forms', Ann. Mat. Pura Appl. (to appear).

[10] L.P. Eisenhart, $A$ treatise on the differential geometry of curves and surfaces (Ginn and Company, Boston, 1909)). (Reprinted as a Dover Phoenix Editions (2004)).

[11] A. Ferrández, 'Riemannian versus Lorentzian submanifolds, some open problems', in Proc. Workshop on Recent Topics in Differential Geometry, Santiago de Compostera 89 (Depto. Geom. y Topologia, Univ. Santiago de Compostera, 1998), pp. 109-130.

[12] A. Ferrández, A. Giménez and P. Lucas, 'Null helices in Lorentzian space forms', Internat J. Modern Phys. A 16 (2001), 4845-4863.

[13] A. Ferrández, A. Giménez and P. Lucas, 'Null generalized helices in Lorentz-Minkowski spaces', J. Phys. A. 35 (2002), 8243-8251.

[14] K. Honda and J. Inoguchi, 'Cesàro's method for Cartan framed null curves', (preprint, 2003).

[15] J. Inoguchi, 'Biharmonic curves in Minkowski 3-space', Int. J. Math. Math. Sci. 21 (2003), $1365-1368$.

[16] J. Inoguchi, 'Biharmonic curves in Minkowski 3-space. Part II', Int. J. Math. Math. Sci. (to appear).

[17] J. Inoguchi, K. Kuwabara and H. Naitoh, 'Grassmann geometry on the 3-dimensional Heisenberg group', Hokkaido Math. J. 34 (2005), 375-391.

[18] J. Inoguchi and S. Lee, 'Null curves in Minkowski 3-space', (preprint).

[19] M.A. Lancret, 'Mémoire sur les courbes à double courbure', Mémoires présentés à l'Institut 1 (1806), 416-454.

[20] B. O'Neill, Elementary differential geometry (Academic Press, New York, London, 1966).

[21] D.J. Struik, Lectures on classical differential geometry (Addison-Wesley Press Inc., Cambridge, MA, 1950). Reprint of the second edition (Dover, New York, 1988). 
[22] S. Tanno, 'Sur une variété de $K$-contact métrique de dimension 3', C. R. Acad. Sci. Paris Ser. A-B 263 (1966), A317-A319.

Department of Mathematics Chonnam National University CNU The Institute of Basic Science Kwangju, 500-757

Korea

e-mail: jtcho@chonnam.ac.kr

Department of Mathematics

Graduate School

Chonnam National University

Kwangju, 500-757

Korea

\author{
Department of Mathematics Education \\ Utsunomiya University \\ Utsunomiya 321-8505 \\ Japan \\ e-mail: inoguchi@cc.utsunomiya-u.ac.jp
}

